VIEWPOINT

\title{
Should antioxidant status be considered in interventional trials with antioxidants?
}

\author{
F Violi, L Loffredo, L Musella, A Marcoccia
}

Heart 2004;90:598-602. doi: 10.1136/hrt.2003.026930

The last decade has seen many trials with antioxidants in patients with cardiovascular disease, with equivocal results. One possible explanation for the disappointing findings is the lack of identification criteria of patients who are potential candidates for antioxidant treatment. Several studies have been carried out in patients at risk of cardiovascular disease, indicating that enhanced oxidative stress is associated with the presence of diabetes, hypercholesterolaemia, hypertension, and smoking. This review analyses the data reported so far to determine whether they clearly support the premise that patients at risk of cardiovascular events may be candidates for antioxidant treatment.

See end of article for authors' affiliations

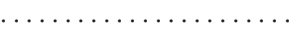

Correspondence to: Professor Francesco Violi, IV Divisione di Clinica Medica, Viale del Policlinico, 155, Roma 00161 , Italy; francesco. violi@uniromal.it
O xidative stress is believed to play a crucial role in the initiation and progression of atherosclerosis disease. Oxidation of low density lipoprotein (LDL) within the vessel wall represents a key step in the accumulation of LDL by resistant macrophages that ultimately become the foam cells of atherosclerotic plaque. ${ }^{1}$ Enzymatic and non-enzymatic oxidation of LDL seems to be involved in this process but its relevance in the evolution of human atherosclerosis is still unclear. ${ }^{1}$ An important consideration is the evident discrepancy between experimental and clinical trials with antioxidants, which have provided divergent results. Most trials with antioxidants in experimental models of atherosclerosis have demonstrated that this treatment is able to retard the progression of atherosclerosis, while the results of clinical trials are conflicting, ${ }^{1}$ inasmuch as positive as well as negative effects have been reported. Investigation of antioxidants for prevention of atherosclerosis stems from observational trials that demonstrated the existence of an inverse relation between the consumption of antioxidant vitamins and the risk of cardiovascular events. However, meta-analysis of the observational studies indicated that among antioxidant vitamins, vitamin $\mathrm{E}$ was the only one that exerted a beneficial effect against atherosclerotic complications. $^{2}$

On the basis of these data almost all the trials have been planned on the assumption that supplementation with vitamin E would represent a useful approach in preventing cardiovascular disease.
However, candidates for antioxidant treatment were not accurately defined probably for the application of the following syllogism:

- oxidant stress is a common pathway for initiation and progression of atherosclerosis

- cardiovascular complications are secondary to atherosclerosis

- all patients with or at risk of cardiovascular complications have enhanced oxidant stress and could obtain beneficial effects from antioxidant treatment.

That this syllogism has been applied to almost all trials with antioxidants is demonstrated by the fact that any patient at risk of cardiovascular events has been indiscriminately enrolled in those trials. We argue on the contrary that, as antioxidant status represents an important marker of oxidative stress, ${ }^{3}$ its determination may be useful in better identifying candidates for antioxidant treatment. In order to substantiate this hypothesis, data inherent to oxidative stress and antioxidant status in patients at risk of cardiovascular disease, and in patients included in observational and interventional trials, have been reviewed. As antioxidant vitamins, such as vitamins $\mathrm{E}$ and $\mathrm{C}$, are major determinants of antioxidant status and have been the object of the most important research in this field, our analysis essentially focuses on the clinical relevance of these vitamins in patients with cardiovascular disease.

\section{DIABETES AND OXIDATIVE STRESS}

Blood analysis of lipid peroxides or measurement of urinary excretion of isoprostanes provided evidence that oxidative stress is enhanced in patients with diabetes. ${ }^{4}$ The impact of these data in the context of atherosclerosis progression is still unclear but there is some evidence supporting a role for oxidative stress in contributing to the deterioration of vascular disease. For instance, an important finding is the demonstration that endothelium dependent vasodilation is reduced in patients with diabetes and that vitamin $\mathrm{C}$ is able to prevent it, thus indicating a role for oxygen-free radicals in reducing the vasodilatory property of endothelium. ${ }^{5}$ Hyperglycaemia may enhance oxidative stress and in turn induce vascular damage via several pathways, including the formation of the advanced glycated end products that are proatherogenic and prothrombotic substances (fig 1, panel C). Furthermore glucose may alter the balance between free radicals such as $\mathrm{O}_{2}{ }^{\circ}$ and nitric oxide (NO) in endothelial cells; thus NO 
exerts its vasodilatory and antioxidant effect unless it is converted to $\mathrm{ONOO}^{-}$by interaction with $\mathrm{O}_{2}{ }^{\circ}$. This deleterious effect occurs in endothelial cells exposed to glucose, which, in fact, favours the formation of $\mathrm{O}_{2}{ }^{\circ}$ and in turn promotes oxidation. ${ }^{\circ}$

An interesting mechanism potentially accounting for enhanced production of reactive oxygen species (ROS) by glucose is reported in fig 1. Hyperglycaemia could enhance endothelial $\mathrm{O} 2^{\circ}$ generation via activation of cyclooxygenase pathway which is known to generate ROS with a mechanism involving $\mathrm{NAD}(\mathrm{P}) \mathrm{H}$ oxidase. ${ }^{7}$ The potential role of this enzyme in inducing oxidative stress has been recently demonstrated by Guzik and colleagues who studied the expression of $\mathrm{NAD}(\mathrm{P}) \mathrm{H}$ oxidase in the vessel wall of diabetic and healthy subjects. ${ }^{8}$ They found that, compared to controls, vascular expression of $\mathrm{NAD}(\mathrm{P}) \mathrm{H}$ oxidase subunits, $\mathrm{p} 22$ phox and p47 phox, were overexpressed in diabetic patients. ${ }^{8}$

\section{HYPERCHOLESTEROLAEMIA AND OXIDATIVE STRESS}

There is experimental and clinical evidence indicating that hypercholesterolemia is associated with enhanced oxidative stress. Oxygen free radicals, such as $\mathrm{O}_{2}{ }^{\circ}$, and F2 isoprostanes, have been found to be elevated in the arteries of hypercholesterolaemic animals or in the urine of patients with high serum cholesterol, respectively. ${ }^{10}$ The relevance of these findings in the context of pathophysiology of atherosclerosis is unclear, although there is some evidence that in this setting oxidative stress may have a role in reducing the vasodilatory property of endothelium. ${ }^{11}$ Conversely, we do not yet have evidence that the increase of these variables actually represents a marker of progression of atherosclerotic disease.

Two hypotheses can be made in explaining why hypercholesterolaemia enhances oxidative stress (fig 1, panels A, B). Cholesterol has been recently shown to activate the metabolism of the arachidonic acid pathway, ${ }^{12}$ which in turn seems to be associated with $\mathrm{NAD}(\mathrm{P}) \mathrm{H}$ oxidase activation.? This hypothesis has been recently underscored by our group showing that platelet incubation with cholesterol enhanced $\mathrm{O}_{2}{ }^{\circ}$ production and that inhibition of PLA2 or NADPH oxidase enzymes significantly reduced $\mathrm{O}_{2}$ formation (fig 1 , panel B). ${ }^{12}$
The cascade of cholesterol biosynthesis may represent per se another pathway leading to enhanced oxidative stress. Intracellular metabolism of mevalonate leads, in fact, to the formation of protein farnesylation, which has a key role in the production of proinflammatory and pro-oxidant cytokines such as tumour necrosis factor $\alpha(\mathrm{TNF} \alpha)$ (fig l, panel A). ${ }^{13}$ In accordance with this, treatment of hypercholesterolaemic patients with an inhibitor of HMG-CoA-reductase was associated with reduced monocyte formation of $\mathrm{TNF} \alpha$, suggesting a relation between cholesterol and intracellular formation of pro-oxidant cytokines. ${ }^{13}$

\section{HYPERTENSION AND OXIDATIVE STRESS}

Experimental studies have provided evidence that oxidative stress is increased in hypertension. The increase in blood pressure may, for example, enhance vascular production of superoxide with a mechanism that is independent of the activation of the renin-angiotensin system. ${ }^{14}$ On the other hand there is experimental evidence supporting the hypothesis that angiotensin converting enzyme system activation is associated with vascular overproduction of $\mathrm{O}_{2}{ }^{0.15}$ It is interesting, however, that both mechanisms have a common pathway for enhancing oxidative stress-that is, activation of NADPH oxidase enzyme (fig 1, panel D). The relation between oxidative stress and hypertension has recently been demonstrated in humans using platelets as a tool for investigating the cellular production of $\mathrm{O}_{2}{ }^{0.16}$ This study suggested that patients with hypertension have enhanced platelet formation of $\mathrm{O}_{2}{ }^{\circ}$ which may be mediated by ATl receptors, and that NADPH-oxidase plays a pivotal role in eliciting angiotensin II induced platelet $\mathrm{O}_{2}{ }^{\circ}$ production. ${ }^{16}$

The clinical relevance of these findings in the context of atherosclerotic disease is unclear, however. Enhanced oxidative stress by the vessel wall could have some role in reducing the vasodilatory property of endothelium. Data regarding this obtained following intravenous infusion of ascorbic acid seem to provide support that oxidative stress could impair arterial vasodilation by inhibiting NO activity. ${ }^{17}$ However, it remains to be established whether patients with hypertension have systemic signs of oxidative stress, and the impact of this phenomenon on the progression of atherosclerotic disease still needs to be ascertained.

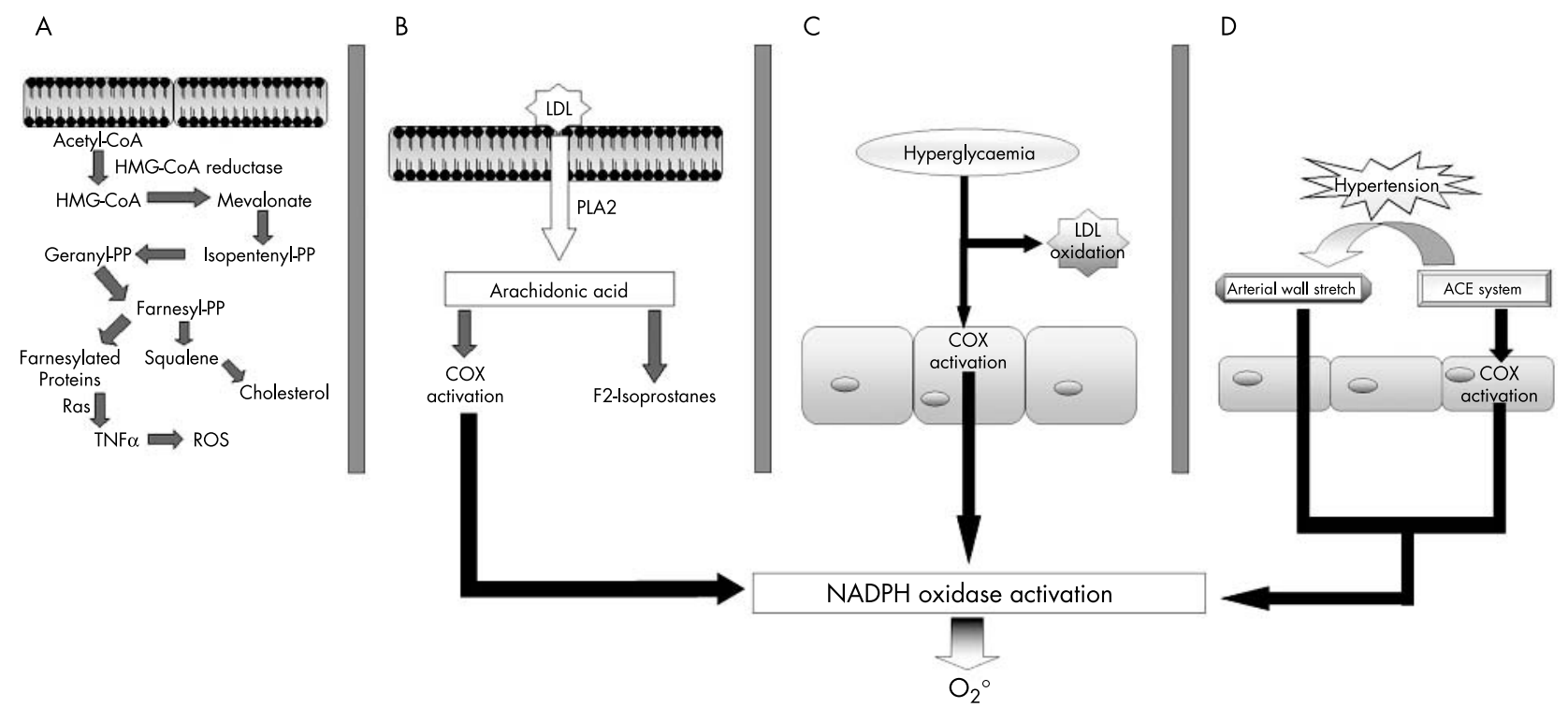

Figure 1 Hypothetical mechanisms that may potentially account for enhanced formation of oxygen-free radicals in hypercholesterolaemia (A and B), hyperglycaemia (C), and hypertension (D). 


\section{SMOKING, OBESITY, AND OXIDATIVE STRESS}

In a study including smokers and non-smokers, Miller and colleagues ${ }^{18}$ measured some markers of oxidative stress after three weeks of following the same diet. Compared to nonsmokers, smokers had significantly higher breath ethane and serum thiobarbituronic acid reactive substances (TBARS). Furthermore, Morrow and colleagues ${ }^{19}$ found that the plasma concentrations and urinary excretion of F2 isoprostane were significantly higher in smokers than in non-smokers and that cessation of smoking was associated with a significant decrease of isoprostanes. ${ }^{19}$

The relation between obesity and oxidative stress has been recently investigated in women with android or gynoid obesity. Obese women had higher oxidative stress than controls; enhanced oxidative stress was increased more in android obesity than in gynoid obesity, suggesting a more pronounced relation between oxidative stress and visceral obesity..$^{20}$

\section{ANTIOXIDANT STATUS IN PATIENTS WITH RISK FACTORS FOR ATHEROSCLEROSIS}

As reported in table 1 , we found few studies ${ }^{21-35}$ that measured the circulating concentrations of vitamins $\mathrm{E}$ and $\mathrm{C}$ in patients with risk factors for atherosclerosis. However, data are extremely variable and difficult to interpret because of lack of control groups. Vitamin $\mathrm{C}$ has been measured in patients with hypertension, diabetes, hypercholesterolaemia, and smoking habit with values ranging from 30-50 $\mu \mathrm{M}$; a large variability of data is particularly observed in smoking and diabetic patients. Dispersion of data is even more evident for vitamin $\mathrm{E}$. Thus, in patients with risk factors for atherosclerosis, vitamin E ranges from 1.6-5.8 $\mu \mathrm{mol} / \mathrm{mmol}$ cholesterol, with large variations within the same category of patients. Apart from hypertensive patients where only one report of plasma vitamin $\mathrm{E}$ was found, the other categories of patients at risk of cardiovascular disease are characterised by a wide variation of data with values also above $5 \mu \mathrm{mol} / \mathrm{mmol}$ cholesterol, which identifies patients at low risk of cardiovascular disease. ${ }^{36}$ The reason for this large variability cannot be explained as yet, but it is possible that oxidative stress may condition the rate of vitamin consumption. This hypothesis may be supported by a previous study showing an inverse correlation between F2 isoprostanes and circulating concentrations of vitamin $\mathrm{E},{ }^{37}$ but we are not certain that elevated markers of oxidative stress are always associated with low antioxidant status. For example, a recent report showed that in animals with cardiovascular aging, which is associated with enhanced oxidative stress, circulating and tissue concentrations of vitamin E were elevated. ${ }^{38}$ Assuming that this might also occur in human atherosclerosis, the evidence of enhanced oxidant stress alone perhaps does not imply the existence of low antioxidant status and justify supplementation with antioxidant vitamins.

Table 1 Mean values of vitamin E/cholesterol ratio and vitamin C plasma values observed in patients at risk of atherosclerotic complications (references are indicated)

\begin{tabular}{lll}
\hline & $\begin{array}{l}\text { Vitamin C plasma } \\
\text { values ( } \mu \mathrm{mol} / \mathrm{I})\end{array}$ & $\begin{array}{l}\text { Vitamin E plasma } \\
\text { values }(\mu \mathrm{mol} / \mathrm{mmol} \\
\text { cholesterol) }\end{array}$ \\
\hline Hypertensive patients & $34.8^{21}-50^{22}$ & $5.0^{23}$ \\
Smokers & $33^{24}-51.8^{25}$ & $1.66^{24}-4.64^{26}$ \\
NIDDM patients & $30.4^{27}-87.5^{28}$ & $3.81^{29}-5.78^{30}$ \\
IDDM patients & $8.45^{31}-63.6^{28}$ & $3.89^{29}-4.24^{32}$ \\
Hypercholesterolaemia & $52.3^{33}-70.7^{34}$ & $4.36^{35}-5.8^{33}$ \\
\hline IDDM, insulin dependent diabetes mellitus; NIDDM, non-IDDM.
\end{tabular}

Unfortunately the majority of previous studies exploring oxidative stress in patients with classic risk factors for atherosclerosis included very little information regarding vitamin E plasma concentrations and antioxidant status in control populations. This limits the clinical usefulness of those studies because we are not certain that, in the case of enhanced oxidative stress, antioxidant treatment is warranted. For example, previous studies reported enhanced urinary excretion of isoprostanes in patients with diabetes and hypercholesterolaemia ${ }^{4}{ }^{10}$ but, as shown in table 2, patients with such risk factors may have values above or below $5 \mu \mathrm{M}$ vitamin $\mathrm{E} / \mathrm{mmol}$ cholesterol. The reason for this wide variation of antioxidant status in patients at risk of cardiovascular disease is unclear, but it should be carefully investigated in order to justify the use of antioxidant supplements in this context.

\section{ANTIOXIDANT STATUS IN STUDIES WITH ANTIOXIDANTS \\ Interventional trials}

Clinical trials with antioxidants have been carried out in patients with or without a previous history of cardiovascular disease. Surrogate end points, such as analysis of atherosclerosis progression, or hard end points, such as vascular death and myocardial infarction, have been examined to evaluate the clinical benefit of antioxidant vitamins. ${ }^{39}$ Most trials with antioxidants used vitamin E probably because epidemiological studies documented that regular consumption of this vitamin reduced the risk of cardiovascular events. ${ }^{36}$ Patient selection of these trials was therefore based on the hypothesis that all patients at risk of cardiovascular disease could benefit from supplementation of this vitamin; therefore many primary and secondary interventional trials, such as the GISSI-prevenzione, the HOPE, and the PPP studies, did not consider the antioxidant status as entry criterion and did not report any data inherent to bioavailability of vitamin E..$^{39}$ The lack of this information makes the results of these trials difficult to interpret, and because the serious issue relating to vitamin E bioavailability has been completely ignored. In fact, we demonstrated that about $30 \%$ of subjects did not have any increase in vitamin E plasma values unless vitamin $\mathrm{E}$ was assumed after food intake. ${ }^{40}$ This finding has been recently supported by Carrol and colleagues, ${ }^{41}$ showing a significant increment of plasma vitamin $\mathrm{E}$ when a supplement was given immediately before a meal.

Among the antioxidant trials, five reported plasma values of vitamin $\mathrm{E}$ in control populations. Patients included in these trials suffered from cardiovascular disease or had classic risk factors for atherosclerosis ${ }^{39}$ or renal insufficiency, ${ }^{39}$ or underwent heart transplantation. ${ }^{39}$ In three of

Table 2 Mean values of vitamin $\mathrm{E} /$ cholesterol ratio observed in patients at risk of atherosclerotic complications (observational studies) and in patients included in interventional trials with vitamin $\mathrm{E}$

\begin{tabular}{ll}
\hline & $\begin{array}{l}\text { Vitamin } \mathrm{E} / \text { cholesterol } \\
\text { ( } \mu \mathrm{mol} / \mathbf{m m o l} \text { ) }\end{array}$ \\
\hline $\begin{array}{l}\text { Observational studies } \\
\text { WHO/MONICA }\end{array}$ & 4.38 \\
Angina pectoris & \\
Interventional studies & 3.66 \\
$\mathrm{CHAOS}^{39}$ & 5.67 \\
$\mathrm{ATBC}^{39}$ & 4.50 \\
$\mathrm{SPACE}^{39}$ & 4.92 \\
Transplant associated $_{\text {atherosclerosis }}^{39}$ & 4.37 \\
$\mathrm{HPS}^{39}$ & 5.69 \\
\hline
\end{tabular}


these trials a combination of vitamins was given: the $\mathrm{ATBC}^{39}$ and the HPS $^{39}$ studies administered vitamins E, C, and $\beta$ carotene, the study including patients undergoing heart transplantation administered vitamins $\mathrm{C}$ and $\mathrm{E}^{36}$ and the CHAOS and the SPACE studies ${ }^{39}$ used natural vitamin $\mathrm{E}$ at dosage of 400-800 IU/day. Vitamin E values of study populations were extremely wide ranging from $5.6 \mu \mathrm{mol} /$ mmol cholesterol (the CHAOS and the HPS studies) to $4.3 \mu \mathrm{mol} / \mathrm{mmol}$ (heart transplant atherosclerosis). Assuming that values of vitamin $\mathrm{E}<5 \mu \mathrm{m} / \mathrm{mmol}$ cholesterol identify patients at risk of cardiovascular disease, ${ }^{39}$ we argue that only three studies included patients with low antioxidant status. Among these trials the ATBC provided negative results while the other two studies demonstrated that vitamin E alone or in combination with vitamin $\mathrm{C}$ significantly reduced cardiovascular events.

The effect of antioxidant vitamins has also been investigated using surrogate end points such as carotid atherosclerotic progression. Salonen and colleagues ${ }^{34}$ demonstrated that a combination of $136 \mathrm{IU}$ vitamin E plus $250 \mathrm{mg}$ of slow release vitamin $\mathrm{C}$ slows down atherosclerotic progression in hypercholesterolaemic patients. It was of note that the treatment was more effective in patients with low baseline values of vitamin C. As cholesterol values were not reported we could not calculate the vitamin E/cholesterol ratio and compare it with the values reported in the other trials.

\section{Observational studies}

Previous studies have attempted to assess whether a relation between antioxidant status and coronary heart disease (CHD) exists. For this purpose, different antioxidants, such as vitamin $\mathrm{E}, \mathrm{C}$, and $\beta$ carotene, have been measured in populations affected or not by CHD. The WHO/MONICA project has been one of the largest studies to analyse the behaviour of these vitamins in populations with different incidence of CHD mortality. ${ }^{39}$ In populations with similar values of serum cholesterol and blood pressure, an inverse correlation between CHD mortality and vitamin E plasma values was observed; conversely, no relation existed between CHD mortality and other vitamins. In areas with low and medium coronary mortality, plasma concentrations of vitamin E were 26-28 $\mu \mathrm{M}$, while at sites with the highest CHD mortality rates plasma values were 20-21.5 $\mu \mathrm{M}$. The authors also estimated that the threshold risk for cardiovascular disease would be $<25 \mu \mathrm{M}$, which in this particular population corresponds to $<4.3 \mu \mathrm{M}$ vitamin $\mathrm{E} / \mathrm{mmol}$ cholesterol (table 2). This finding is consistent with other studies showing an inverse correlation between vitamin E plasma values and cardiovascular mortality. ${ }^{36}$ It was noticed, in particular, that in patients with a high risk of cardiovascular mortality, the vitamin E/cholesterol ratio was 3.5, while in those with low risk, the ratio was almost $5{ }^{42}$

The inverse correlation between vitamin E concentrations and CHD was also noted in another observational study in which 110 patients with angina were compared to 394 controls (table 2)..$^{43}$ The study demonstrated that patients with a history of angina had a lower vitamin E/cholesterol ratio than controls $(3.66 v 3.86 \mu \mathrm{mol} / \mathrm{mmol}, \mathrm{p}<0.01)$, with a significant adjusted odds ratio for angina between patients in the lowest and highest quartile.

These findings have been further corroborated by another study in which 102 apparently healthy subjects were followed up for 47.4 months. ${ }^{44}$ This study showed a higher risk of cardiovascular events in subjects in the lowest quartile of vitamin E plasma values compared to those in the highest. Taken together these data suggest that vitamin $\mathrm{E}$ is an important predictor of CHD and may represent an independent risk factor for atherosclerosis and its complication. Because of the lack of standardisation and a somewhat large dispersion of vitamin E/cholesterol ratio values, accurate analysis of vitamin E concentrations in patients and healthy subjects is crucial in order to have a reliable use of this variable in clinical practice and interventional trials. Recently, for example, healthy subjects ${ }^{45}$ were shown to have values of vitamin $\mathrm{E}$ of $3.6 \mu \mathrm{mmol} / \mathrm{mmol}$ cholesterol, which is much less than values reported in control populations. ${ }^{46}$ This finding therefore raises serious concerns regarding the methodology used for measuring vitamins and strongly suggests the need to standardise the assay.

\section{CONCLUSION}

On the basis of these considerations we can conclude that there is compelling evidence that enhanced oxidative stress is detectable in patients with classic risk factors for atherosclerosis, but its impact in the context of atherosclerosis progression is still unclear. The reason for this uncertainty is due to the lack of a clear prospective study indicating that markers of oxidative stress, such as blood lipid peroxides or urinary F2 isoprostane, are of value in predicting the progression of atherosclerosis, although there is evidence suggesting that antibodies against oxidised LDL may be of some utility. ${ }^{47}$ Conversely, epidemiologic studies seem to indicate that low antioxidant status increases the risk of cardiovascular disease. Clinical characteristics of patients with low antioxidant status have not been defined and should be studied in the near future. So far clinical trials with antioxidants have included patients without evaluating either oxidative stress or antioxidant status, and such indiscriminate enrolment could perhaps account for the negative results of antioxidant trials recently emphasised by a meta-analysis. ${ }^{48}$ However, future trials with antioxidants should not be discouraged, but better identification of criteria identifying potential candidates for antioxidant treatment should be studied.

\section{Authors' affiliations}

F Violi, L Loffredo, L Musella, A Marcoccia, IV Divisione di Clinica

Medica, Viale del Policlinico, University "La Sapienza", Roma, Italy

\section{REFERENCES}

1 Steinberg D, Witztum JL. Is the oxidative modification hypothesis relevant to human atherosclerosis? Do the antioxidant trials conducted to date refute the hypothesis? Circulation 2002;105:2107-11.

2 Jha $\mathrm{P}$, Flather $\mathrm{M}$, Lonn $\mathrm{E}$, et al. The antioxidant vitamins and cardiovascular disease. A critical review of epidemiologic and clinical trial data. Ann Intern Med 1995; 123:860-72.

3 Violi F, Micheletta F, luliano L. How to select patient candidates for antioxidant treatment? Circulation 2002;106:e195.

4 Mezzetti A, Cipollone F, Cuccurullo F. Oxidative stress and cardiovascular complications in diabetes: isoprostanes as new markers on an old paradigm. Cardiovasc Res 2000;47:475-88.

5 Timimi FK, Ting HH, Haley EA, et al. Vitamin C improves endotheliumdependent vasodilation in patients with insulin-dependent diabetes mellitus. J Am Coll Cardiol 1998:31:552-7.

6 Cosentino F, Hishikawa K, Katusic ZS, et al. High glucose increases nitric oxide synthase expression and superoxide anion generation in human aortic endothelial cells. Circulation 1997;96:25-8.

7 Wolin MS. Interactions of oxidants with vascular signaling systems. Arterioscler Thromb Vasc Biol 2000;20:1430-42.

8 Guzik TJ, Mussa S, Gastaldi D, et al. Mechanisms of increased vascular superoxide production in human diabetes mellitus: role of $\mathrm{NAD}(\mathrm{P}) \mathrm{H}$ oxidase and endothelial nitric oxide synthase. Circulation 2002;105:1656-62.

9 Ohara Y, Peterson TE, Sayegh HS, et al. Dietary correction of hypercholesterolemia in the rabbit normalizes endothelial superoxide anion production. Circulation 1995;92:898-903.

10 Davì G, Alessandrini P, Mezzetti A, et al. In vivo formation of 8-epiprostaglandin F2 $\alpha$ is increased in hypercholesterolemia. Arterioscler Thromb Vasc Biol 1997; 17:3230-35.

11 Ting HH, Timimi FK, Haley EA, et al. Vitamin C improves endotheliumdependent vasodilation in forearm resistance vessels of humans with hypercholesterolemia. Circulation 1997;95:2617-22.

12 Sanguigni V, Pignatelli P, Caccese D, et al. Increased superoxide anion production by platelets in hypercholesterolemic patients. Thromb Haemost 2002;87:796-801. 
13 Ferro D, Parrotto S, Basili S, et al. Simvastatin inhibits the monocyte expression of proinflammatory cytokines in patients with hypercholesterolemia. J Am Coll Cardiol 2000;36:427-31.

14 Beswick RA, Dorrance AM, Leite R, et al. NADH/NADPH oxidase and enhanced superoxide production in the mineralocorticoid hypertensive rat. Hypertension 2001;38:1107-11.

15 Berry C, Hamilton CA, Brosnan MJ, et al. Investigation into the sources of superoxide in human blood vessels: angiotensin II increases superoxide production in human internal mammary arteries. Circulation 2000;101:2206-12.

16 Sanguigni V, Germanò G, Pignatelli P, et al. Role of ATI receptors in enhancing oxidative stress in patients with hypertension. AHA Scientific Sessions, 2002, Abstract ID, 102176

17 Taddei S, Virdis A, Ghiadoni L, et al. Vitamin C improves endotheliumdependent vasodilation by restoring nitric oxide activity in essential hypertension. Circulation 1998;97:2222-9.

18 Miller ER, Appel $\sqcup$, Jiang $L$, et al. Association between cigarette smoking and lipid peroxidation in a controlled feeding study. Circulation 1997; 96:1097-101

19 Morrow JD, Frei B, Longmire AW, et al. Increase in circulating products of lipid peroxidation (F2-isoprostanes) in smokers. Smoking as a cause of oxidative damage. N Engl J Med 1995;332:1198-203.

20 Davi G, Guagnano MT, Ciabattoni G, et al. Platelet activation in obese women: role of inflammation and oxidant stress. JAMA 2002;288:2008-14.

21 Wen Y, Killalea S, McGettigan P, et al. Lipid peroxidation and antioxidant vitamins $C$ and $E$ in hypertensive patients. Ir J Med Sci 1996;165:210-2.

22 Duffy SJ, Gokce N, Holbrook M, et al. Effect of ascorbic acid treatment on conduit vessel endothelial dysfunction in patients with hypertension. Am J Physiol Heart Circ Physiol 2001;280:H528-34.

23 Pierdomenico SD, Costantini F, Bucci A, et al. Low-density lipoprotein oxidation and vitamins $E$ and $C$ in sustained and white-coat hypertension. Hypertension 1998;31:621-6.

24 Liu CS, Chen HW, Lii CK, et al. Alterations of small-molecular-weight antioxidants in the blood of smokers. Chem Biol Interact 1998;116:143-54

25 Tsuchiya M, Asada A, Kasahara E, et al. Smoking a single cigarette rapidly reduces combined concentrations of nitrate and nitrite and concentrations of antioxidants in plasma. Circulation 2002;105:1155-7.

26 Mol MJ, de Rijke YB, Demacker PN, et al. Plasma levels of lipid and cholestero oxidation products and cytokines in diabetes mellitus and cigarette smoking: effects of vitamin $E$ treatment. Atherosclerosis 1997; 129:169-76.

27 Sinclair AJ, Taylor PB, Lunec J, et al. Low plasma ascorbate levels in patients with type 2 diabetes mellitus consuming adequate dietary vitamin C. Diabet Med 1994;11:893-8.

28 Maxwell SR, Thomason H, Sandler D, et al. Antioxidant status in patients with uncomplicated insulin-dependent and non-insulin-dependent diabetes mellitus. Eur J Clin Invest 1997;27:484-90.

29 Leonhardt W, Hanefeld M, Muller G, et al. Impact of concentrations of glycated hemoglobin, alpha-tocopherol, copper, and manganese on oxidation of low-density lipoproteins in patients with type I diabetes, type II diabetes and control subjects. Clin Chim Acta 1996;254:173-86.

30 Gòde $\mathbf{P}$, Poulsen HE, Parving HH, et al. Double-blind, randomised study of the effect of combined treatment with vitamin $C$ and $E$ on albuminuria in type 2 diabetic patients Diabet Med 2001;18:756-60.
31 Seghieri G, Martinoli L, di Felice M, et al. Plasma and platelet ascorbate pools and lipid peroxidation in insulin-dependent diabetes mellitus. Eur J Clin Invest 1998;28:659-63.

32 Jain SK, McVie R, Jaramillo JJ, et al. The effect of modest vitamin E supplementation on lipid peroxidation products and other cardiovascular risk factors in diabetic patients. Lipids 1996:31(suppl):S87-90

33 Porkkala-Sarataho E, Nyyssonen K, Salonen JT. Increased oxidation resistance of atherogenic plasma lipoproteins at high vitamin $E$ levels in nonvitamin E supplemented men. Atherosclerosis 1996;124:83-94.

34 Salonen RM, Nyyssonen K, Kaikkonen J, et al. Antioxidant supplementation in atherosclerosis prevention study. Six-year effect of combined vitamin $C$ and $E$ supplementation on atherosclerotic progression: the antioxidant supplementation in atherosclerosis prevention (ASAP) study. Circulation 2003; 107:947-53.

35 Nourooz-Zadeh J, Smith CCT, Betteridge DJ. Measures of oxidative stress in heterozygous familial hypercholesterolaemia. Atherosclerosis $2001 ; 156: 435-41$.

36 Pryor WA. Vitamin E and heart disease: basic science to clinical intervention trials. Free Radic Biol Med 2000;28:141-64.

37 Pratico D, Tangirala RK, Rader DJ, et al. Vitamin E suppresses isoprostane generation in vivo and reduces atherosclerosis in ApoE-deficient mice. Nat Med 1998;4:1 189-92.

38 Van der Loo B, Labugger R, Abeischer CC. Cardiovascular aging is associated with vitamin $\mathrm{E}$ increase. Circulation 2002:105:1635-8.

39 Gotto AM. Antioxidants, statins, and atherosclerosis. J Am Coll Cardiol 2003;41:1205-10

40 luliano L, Micheletta F, Maranghi M, et al. Bioavailability of vitamin E as function of food intake in healthy subjects: effects on plasma peroxidescavenging activity and cholesterol-oxidation products. Arterioscler Thromb Vasc Biol 2001;21:E34-7.

41 Carroll MF, Schade DS. Timing of antioxidant vitamin ingestion alters postprandial proatherogenic serum markers. Circulation 2003;108:24-31.

42 Gey KF. Vitamin E and other essential antioxidants regarding coronary heart disease: risk assessment studies. Epidemiological basis of the antioxidant hypothesis of cardiovascular disease. In: Packer L, Fuchs J, eds. Vitamin E in health and disease. New York: Marcel Dekker, Inc, 1993:589-633.

43 Riemersma RA, Wood DA, Macintyre CC, et al. Risk of angina pectoris and plasma concentration of vitamins $\mathrm{A}, \mathrm{C}$, and $\mathrm{E}$ and carotene. Lancet 1991;337:1-5.

44 Mezzetti A, Zuliani G, Romano F, et al. Vitamin E and lipid peroxide plasma levels predict the risk of cardiovascular events in a group of healthy very old people. J Am Geriatr Soc 2001:49:533-7.

45 Hodis HN, Mack WJ, La Bree L, et al, for VEAPS Research Group. Alphatocopherol supplementation in healthy individuals reduces low-density lipoprotein oxidation but not atherosclerosis: the vitamin $\mathrm{E}$ atherosclerosis prevention study (VEAPS). Circulation 2002;106:1453-9.

46 Violi F, Micheletta F, luliano L. Vitamin E supplementation. Lancet 2000;357:632-3.

47 Inove T, Uchida T, Kamishirado H, et al. Antibody against oxidized low density lipoprotein may predict progression or regression of atherosclerotic coronary artery disease. J Am Coll Cardiol 2001;37:1871-6.

48 Vivekananthan DP, Penn MS, Sapp SK, et al. Use of antioxidant vitamins for the prevention of cardiovascular disease: meta-analysis of randomised trials. Lancet 2003;361:2017-23.

\section{ELECTRONIC PAGES}

\section{Heart Online case reports: www.heartjnl.com}

tro he follow electronic only articles are published in conjunction with this issue of Heart.

\section{Right ventricular diastolic dysfunction and patent foramen ovale causing profound cyanosis}

H Shnaider, A Shiran, A Lorber

A 73 year old woman presented with profound central cyanosis and a history of a minor stroke. She had normal heart morphology, normal pulmonary artery pressure, and a normal coronary angiography. A patent foramen ovale (PFO) with a massive right to left shunt was demonstrated at the atrial level, with normal pulmonary venous saturations and $\mathrm{PO}_{2}$ values. This rare, age related case of right ventricular diastolic dysfunction in a normotensive patient revealed a generous PFO allowing a pronounced right to left shunt. $6 / \mathrm{e} 31$

\section{Human coronary morphology after $\beta$ radiation brachytherapy of in-stent restenosis P H Grewe, T Deneke, C Hanefeld, K-M Müller}

This case report discusses the human coronary morphological findings 18 hours after brachytherapy ( $\beta$ radiation) of an instent restenosis. Brachytherapy produced aseptic inflammation of the periadventitial connective tissue integrating the vasa vasorum in the acute phase. The stent neointima eight months after stenting and acutely 18 hours after radiation consisted of the same cellular components as human stent neointima of specimen not additionally treated with radiation. No evidence of necrosis or excessive fibrotic alterations of the arterial vessel wall have been found.

(Heart 2004;90:e32) www.heartjnl.com/cgi/content/full/90/ $6 / \mathrm{e} 32$ 